\title{
Extragradient Method for Solutions of Variational Inequality Problems in Banach Spaces
}

\author{
H. Zegeye ${ }^{1}$ and N. Shahzad ${ }^{2}$ \\ ${ }^{1}$ Department of Mathematics, University of Botswana, Private Bag 00704, Gaborone, Botswana \\ ${ }^{2}$ Department of Mathematics, King Abdulaziz University, P.O. Box 80203, Jeddah 21589, Saudi Arabia \\ Correspondence should be addressed to N. Shahzad; nshahzad@kau.edu.sa
}

Received 27 March 2013; Accepted 11 May 2013

Academic Editor: Ru Dong Chen

Copyright (c) $2013 \mathrm{H}$. Zegeye and N. Shahzad. This is an open access article distributed under the Creative Commons Attribution License, which permits unrestricted use, distribution, and reproduction in any medium, provided the original work is properly cited.

We introduce an iterative process which converges strongly to solutions of a certain variational inequity problem for $\eta$-inverse strongly accretive mappings in the set of common fixed points of finite family of strictly pseudocontractive mappings in Banach spaces. Our theorems improve and unify most of the results that have been proved for this important class of nonlinear operators.

\section{Introduction}

Let $E$ be a real normed linear space with dual $E^{*}$. For $1<q<$ $\infty$, we denote by $J_{q}$ the generalized duality mapping from $E$ to $2^{E^{*}}$ defined by

$$
J_{q}(x):=\left\{x^{*} \in E^{*}:\left\langle x, x^{*}\right\rangle=\|x\|^{q},\left\|x^{*}\right\|=\|x\|^{q-1}\right\},
$$

where $\langle\cdot, \cdot\rangle$ denotes the duality pairing. In particular, $J=J_{2}$ is called the normalized duality map. It is well known (see e.g., [1]) that $J_{q}$ is single valued if $E$ is smooth and that

$$
J_{q}(x)=\|x\|^{q-2} J(x), \quad x \neq 0 .
$$

In the sequel, we will denote the single-valued generalized map by $j_{q}$.

A mapping $A$ with domain $D(A) \subseteq E$ and range $R(A)$ in $E$ is called $\alpha$-strongly accretive if there exist $\alpha \in(0,1)$ and $j_{q}(x-y) \in J_{q}(x-y)$ such that

$$
\left\langle A x-A y, j_{q}(v-u)\right\rangle \geq \alpha\|x-y\|^{q} .
$$

$A$ is called $\eta$-inverse strongly accretive if there exist $\eta \in(0,1)$ and $j_{q}(x-y) \in J_{q}(x-y)$ such that

$$
\begin{array}{r}
\left\langle A x-A y, j_{q}(x-y)\right\rangle \geq \eta\|A x-A y\|^{q}, \\
\text { for every } x, y \in D(A) .
\end{array}
$$

Let $C$ be a nonempty, closed, and convex subset of $E$ and, let $A: C \rightarrow E$ be a nonlinear mapping. The variational inequality problem is to

$$
\text { find } u \in C \text { such that }\langle A u, j(v-u)\rangle \geq 0, \quad \forall v \in C,
$$

for some $j(v-u) \in J(v-u)$. The set of solutions of variational inequality problem is denoted by $\operatorname{VI}(C, A)$. If $E:=H$, a real Hilbert space, the variational inequality problem reduces to

$$
\text { find } u \in C \text { such that }\langle A u, v-u\rangle \geq 0, \quad \forall v \in C \text {, }
$$

which was introduced and studied by Stampacchia [2].

Variational inequality theory has emerged as an important tool in studying a wide class of related problems arising in mathematical, physical, regional, engineering, and nonlinear optimization sciences (see, for instance, [3-12]).

In 1976, Korpelevič [4] introduced the following wellknown extragradient method:

$$
\begin{aligned}
y_{n} & =P_{C}\left(x_{n}-\gamma A x_{n}\right), \\
x_{n+1} & =P_{C}\left(x_{n}-\gamma A y_{n}\right), \quad n \geq 0,
\end{aligned}
$$

where $P_{C}$ is the metric projection from $\mathbb{R}^{n}$ onto its subset $C$, for some $\gamma>0$, and $A: C \rightarrow \mathbb{R}^{n}$ is an accretive operator. He proved that the sequence $\left\{x_{n}\right\}$ converges to a solution of the variational inequality (6). 
Furthermore, Noor [6] proved that the iterative scheme, given by

$$
\begin{aligned}
y_{n} & =P_{C}\left(x_{n}-\gamma A x_{n}\right), \\
x_{n+1} & =P_{C}\left(y_{n}-\gamma A y_{n}\right), \quad n \geq 0,
\end{aligned}
$$

where $A: C \subseteq \mathbb{R}^{n} \rightarrow \mathbb{R}^{n}$ is an accretive operator, converges to a solution of the variational inequality (6).

We note that the above algorithms give strong convergence to a solution of the variational inequality (6). However, both algorithms fail, in general, to converge strongly in the setting of infinite-dimensional Hilbert spaces.

In 2006, Aoyama et al. [13] introduced and studied the following iterative algorithm in a uniformly convex and 2-uniformly smooth Banach spaces possessing weakly sequentially continuous duality mapping:

$$
x_{n+1}=\alpha_{n} x_{n}+\left(1-\alpha_{n}\right) Q_{C}\left[x_{n}-\lambda_{n} A x_{n}\right], \quad n \geq 0,
$$

where $Q_{C}$ is a sunny nonexpansive retraction from $E$ onto a closed and convex $C, A: C \rightarrow E$ is an $\eta$-inverse strongly accretive mapping and $\left\{\alpha_{n}\right\}$ and $\left\{\lambda_{n}\right\}$ subsets of real numbers, satisfy certain conditions. They proved that the sequence in (9) converges weakly to a point $z \in V(C, A)$.

Recently, Yao et al. [8] introduced and considered the following iterative method for $\eta$-strongly accretive mappings in a uniformly convex and 2-uniformly smooth Banach space possessing weakly sequentially continuous duality mapping:

$$
\begin{gathered}
y_{n}=Q_{C}\left(x_{n}-\lambda_{n} A x_{n}\right), \\
x_{n+1}=\alpha_{n} u+\beta_{n} x_{n}+\gamma_{n} Q_{C}\left(y_{n}-\lambda_{n} A y_{n}\right), \quad n \geq 0,
\end{gathered}
$$

where $Q_{C}$ is a sunny nonexpansive retraction from $E$ onto $C$. They proved that the sequence $\left\{x_{n}\right\}$ defined by (10) converges strongly to $Q_{V I(C, A)} u$ provided that real sequences $\left\{\alpha_{n}\right\},\left\{\beta_{n}\right\}$, $\left\{\gamma_{n}\right\}$, and $\left\{\lambda_{n}\right\}$ satisfy certain conditions.

Let $C$ be a nonempty subset of a real Banach space $E$. A mapping $T: C \rightarrow E$ is called $\lambda$-strictly pseudocontractive of Browder-Petryshyn type [14] if for all $x, y \in D(T)$ there exist $\lambda>0$ and $j_{q}(x-y) \in J_{q}(x-y)$ such that

$$
\left\langle T x-T y, j_{q}(x-y)\right\rangle \leq\|x-y\|^{q}-\lambda\|x-y-(T x-T y)\|^{q} .
$$

$T$ is called Lipschitz if there exists $L \geq 0$ such that

$$
\|T x-T y\| \leq L\|x-y\| \quad \forall x, y \in D(T) .
$$

If $L<1$ in (12), then $T$ is called contraction, while $T$ is said to be nonexpansive if $L=1$.

If $E=H$, a real Hilbert space, then (11) is equivalent to the inequality

$$
\begin{aligned}
\|T x-T y\|^{2} \leq & \|x-y\|^{2} \\
& +k\|x-y-(T x-T y)\|^{2}, \quad k=(1-2 \lambda),
\end{aligned}
$$

and we can assume also that $k \geq 0$, so that $k \in[0,1)$. A point $x \in C$ is a fixed point of $T$ if $T x=x$, and we denote by $F(T)$ the set of fixed points of $T$; that is, $F(T)=\{x \in C: T x=x\}$.

In 2001, Yamada [7] introduced a hybrid steepest descent method which relates solutions of variational inequality problems with fixed point of mappings in Hilbert spaces. He proved that if $T$ is nonexpansive self-map on $C$ and $A$ is an $\eta$ strongly accretive mapping from $C$ into $E$ satisfying certain conditions, then the sequence defined by

$$
x_{n+1}=T x_{n}-\mu \lambda_{n} A\left(T x_{n}\right), \quad n \geq 0,
$$

converges strongly to the unique solution of the variational inequality

$$
\begin{aligned}
& \text { Find } x^{*} \in F(T) \text { such that }\left\langle A x^{*}, x-x^{*}\right\rangle \geq 0 \text {, } \\
& \forall x \in F(T) \text {. }
\end{aligned}
$$

The above results naturally bring us to the following question.

Question. Could we produce an iterative scheme which approximates a solution of variational inequality (5) for $\eta$ inverse strongly accretive mappings in Banach spaces?

In this paper, motivated by Yao et al. [8] and Yamada [7], it is our purpose to introduce an iterative scheme which converges strongly to a solution of the variational inequality (5) for $\eta$-inverse strongly accretive mapping in the set of common fixed points of finite family of strictly pseudocontractive mappings in a uniformly convex and $q$-uniformly smooth Banach space $E$ possessing weakly sequentially continuous duality mapping. Our results complement or improve the results of Yao et al. [8], Aoyama et al. [13], and some authors.

\section{Preliminaries}

Let $E$ be a real Banach space. The modulus of smoothness of $E$ is the function $\rho_{E}:[0, \infty) \rightarrow[0, \infty)$ defined by $\rho_{E}(\tau):=$ $\sup \{(1 / 2)(\|x+y\|+\|x-y\|)-1:\|x\|=1,\|y\|=\tau\}$. If $\rho_{E}(\tau)>0$ for all $\tau>0$, then $E$ is said to be smooth. If there exists a constant $c>0$ and a real number $1<q<\infty$, such that $\rho_{E}(\tau) \leq c \tau^{q}$, then $E$ is said to be q-uniformly smooth.

If $E$ is a real $q$-uniformly smooth Banach space, then by [15], the following geometric inequality holds:

$$
\|x+y\|^{q} \leq\|x\|^{q}+q\left\langle y, j_{q}(x)\right\rangle+c_{q}\|y\|^{q},
$$

for all $x, y \in E$ and some real constant $c_{q}>0$.

It is well known (see e.g., [16]) that

$$
L_{p}\left(l_{p}\right) \text { or } W_{m}^{p} \text { is } \begin{cases}p \text {-uniformly smooth } & \text { if } 1<p<2, \\ 2 \text {-uniformly smooth } & \text { if } p \geq 2 .\end{cases}
$$

The Banach space $E$ is said to be uniformly convex if, given $\varepsilon>0$, there exists $\delta>0$, such that, for all $x, y \in E$ with $\|x\| \leq 1,\|y\| \leq 1$ and $\|x-y\| \geq \varepsilon,\|(1 / 2)(x+y)\| \leq 1-\delta$. It is well known that $L_{p}, \ell_{p}$, and Sobolev spaces $W_{m}^{p},(1<p<\infty)$ are uniformly convex.

Let $C \subseteq E$ be closed convex and $Q$ a mapping of $E$ onto $C$. Then, $Q$ is said to be sunny if $Q(Q(x)+t(x-Q(x)))=Q(x)$ 
for all $x \in E$ and $t \geq 0$. A mapping $Q$ of $E$ into $C$ is said to be a retraction if $Q^{2}=Q$. If a mapping $Q$ is a retraction, then $Q(z)=(z)$ for every $z \in R(Q)$, range of $Q$. A subset $C$ of $E$ is said to be a sunny nonexpansive retract of $E$ if there exists a sunny nonexpansive retraction of $E$ onto $C$, and it is said to be a nonexpansive retract of $E$ if there exists a nonexpansive retraction of $E$ onto $C$. If $E=H$, the metric projection $P_{C}$ is a sunny nonexpansive retraction from $H$ to any closed convex subset of $H$. Moreover, if $C$ is a nonempty closed convex subset of a uniformly convex and uniformly smooth real Banach space $E$ and $T$ is a nonexpansive mapping of $C$ into itself with $F(T) \neq \emptyset$, then the set $F(T)$ is a sunny nonexpansive retract of $C$.

In what follows, we will make use of the following lemmas.

Lemma 1 (see, e.g., [17]). Let E be a smooth Banach space, and let $K$ be a nonempty subset of $E$. Let $Q: E \rightarrow K$ be a retraction, and let $J$ be the normalized duality map on $E$. Then, the following are equivalent:

(i) $Q$ is sunny nonexpansive,

(ii) $\langle x-Q(x), J(y-Q(x))\rangle \leq 0$ for all $x \in E$ and $y \in K$.

Lemma 2 (see [18]). Let $\left\{a_{n}\right\}$ be a sequence of nonnegative real numbers satisfying the following relation:

$$
a_{n+1} \leq\left(1-\alpha_{n}\right) a_{n}+\alpha_{n} \delta_{n}, \quad n \geq n_{0},
$$

where $\left\{\alpha_{n}\right\} \subset(0,1)$ and $\left\{\delta_{n}\right\} \subset \mathbb{R}$ satisfying the following conditions: $\lim _{n \rightarrow \infty} \alpha_{n}=0, \sum_{n=1}^{\infty} \alpha_{n}=\infty$, and lim $\sup _{n \rightarrow \infty} \delta_{n} \leq 0$. Then, $\lim _{n \rightarrow \infty} a_{n}=0$.

Lemma 3 (see [13]). Let $C$ be a nonempty closed convex subset of a smooth Banach space E. Let $Q_{C}$ be a sunny nonexpansive retraction from $E$ onto $C$, and let $A$ be an accretive operator of $C$ into $E$. Then, for all $\lambda>0$,

$$
V I(C, A)=F\left(Q_{C}(I-\lambda A)\right),
$$

where $\operatorname{VI}(C, A)=\left\{x^{*} \in C:\left\langle A x^{*}, J\left(x-x^{*}\right)\right\rangle \geq 0, \forall x \in C\right\}$.

Lemma 4 (see [17]). Let $C$ be a nonempty bounded closed convex subset of a uniformly convex Banach space $E$, and let $T$ be nonexpansive mapping of $C$ into itself. If $\left\{x_{n}\right\}$ is a sequence of $C$ such that $x_{n} \rightarrow x$ weakly and $x_{n}-T x_{n} \rightarrow 0$ strongly, then $x$ is a fixed point of $T$.

Lemma 5 (see [19]). Let E be a real Banach space. Then, for any given $x, y \in E$, the following inequality holds:

$$
\|x+y\|^{2} \leq\|x\|^{2}+2\langle y, j(x+y)\rangle, \quad \forall j(x+y) \in J(x+y) .
$$

Lemma 6 (see [20]). Let $K$ be a nonempty closed convex subset of a strictly convex Banach space E. Let $T_{i}: K \rightarrow E, i=$ $1,2, \ldots, r$, be a family of nonexpansive mappings such that $\bigcap_{i=1}^{r} F\left(T_{i}\right) \neq \emptyset$. Let $\alpha_{0}, \alpha_{1}, \alpha_{2}, \ldots, \alpha_{r}$ be real numbers in $(0,1)$ such that $\sum_{i=0}^{r} \alpha_{i}=1$, and let $T:=\alpha_{0} I+\alpha_{1} T_{1}+\cdots+\alpha_{r} T_{r}$. Then, $T$ is nonexpansive, and $F(T)=\bigcap_{i=1}^{r} F\left(T_{i}\right)$.
Lemma 7 (see [21]). Let $C$ be a nonempty, closed and convex subset of a real uniformly convex and smooth Banach space $E$. Let $T_{i}: C \rightarrow E, i=1, \ldots, N$, be $\lambda_{i}$-strictly pseudocontractive mappings such that $\bigcap_{i=1}^{N} F\left(T_{i}\right) \neq \emptyset$. Let $T:=\theta_{1} T_{1}+\theta_{2} T_{2}+$ $\cdots+\theta_{N} T_{N}$ with $\theta_{1}+\theta_{2}+\cdots+\theta_{N}=1$. Then $T$ is $\lambda$-strictly pseudocontractive with $\lambda:=\min \left\{\lambda_{i}: i=1, \ldots, N\right\}$ and $F(T)=\bigcap_{i=1}^{N} F\left(T_{i}\right)$.

Lemma 8 (see [22]). Let $C$ be a nonempty closed and convex subset of a real q-uniformly smooth Banach space $E$ for $1<q<$ $\infty$. Let $T: C \rightarrow E$ be a $\lambda$-strictly pseudocontractive mapping. Then, for $0<\mu<\mu_{0}=\min \left\{1,\left(q \lambda / c_{q}\right)^{1 /(q-1)}\right\}$, where $L$ is the Lipchitz constant of $T$ and $c_{q}$ is a constant in (16), the mapping $T_{\mu} x:=(1-\mu) x+\mu T x$ is nonexpansive, and $F\left(T_{\mu}\right)=F(T)$.

Lemma 9. Let $C$ be a nonempty closed and convex subset of a a real q-uniformly smooth Banach space $E$ for $1<q<\infty$. Let $A: C \rightarrow E$ be an $\eta$-inverse strongly accretive mapping. Then, for $0<\gamma<\left(q \eta / c_{q}\right)^{1 /(q-1)}$, the mapping $A_{\mu} x:=(x-\gamma A x)$ is nonexpansive.

Proof. Now, using inequality (16), we get that

$$
\begin{aligned}
\| A_{\gamma} x & -A_{\gamma} y \|^{q} \\
= & \|(x-y)-\gamma(A x-A y)\|^{q} \\
\leq & \|x-y\|^{q}-q \gamma\left\langle A x-A y, j_{q}(x-y)\right\rangle \\
& +\gamma^{q} c_{q}\|A x-A y\|^{q} \\
\leq & \|x-y\|^{q}-q \gamma \eta\|A x-A y\|^{2}+\gamma^{q} c_{q}\|A x-A y\|^{2} \\
\leq & \|x-y\|^{q}-\gamma\left(q \eta-\gamma^{q-1} c_{q}\right)\|A x-A y\|^{q}, \\
\leq & \|x-y\|^{q} .
\end{aligned}
$$

The proof is complete.

Lemma 10 (see [10]). Let $E$ be a uniformly convex Banach space, and let $B_{R}(0)$ be a closed ball of $E$. Then, there exists a continuous strictly increasing convex function $g:[0, \infty) \rightarrow$ $[0, \infty)$ with $g(0)=0$ such that

$$
\begin{aligned}
\| \alpha_{0} x_{0} & +\alpha_{1} x_{1}+\alpha_{2} x_{2}+\cdots+\alpha_{k} x_{k} \|^{2} \\
& \leq \sum_{i=0}^{k} \alpha_{i}\left\|x_{i}\right\|^{2}-\alpha_{s} \alpha_{t} g\left(\left\|x_{s}-x_{t}\right\|\right),
\end{aligned}
$$

for $x_{i} \in B_{R}(0):=\{x \in E:\|x\| \leq R\}, i=0,1,2, \ldots, k$ with $\sum_{i=0}^{k} \alpha_{i}=1$.

Lemma 11 (see [5]). Let $\left\{a_{n}\right\}$ be sequences of real numbers such that there exists a subsequence $\left\{n_{i}\right\}$ of $\{n\}$ such that $a_{n_{i}}<a_{n_{i}+1}$, for all $i \in \mathbb{N}$. Then, there exists a nondecreasing sequence $\left\{m_{k}\right\} \subset \mathbb{N}$ such that $m_{k} \rightarrow \infty$, and the following properties are satisfied by all (sufficiently large) numbers $k \in \mathbb{N}$ :

$$
a_{m_{k}} \leq a_{m_{k}+1}, \quad a_{k} \leq a_{m_{k}+1} .
$$

In fact, $m_{k}=\max \left\{j \leq k: a_{j}<a_{j+1}\right\}$. 


\section{Main Results}

Let $C$ be a nonempty closed convex subset of a real uniformly convex and $q$-uniformly smooth Banach space $E(1<q<$ $\infty)$. Let $T_{i}: C \rightarrow C$, for $i=1, \ldots, N$, be a $\lambda_{i}$-strictly pseudocontractive mappings, and let $A: C \rightarrow E$ be an $\eta$ inverse strongly accretive mapping. Then, in what follows, we will study the variational inequality

Find $x^{*} \in \cap_{i=1}^{N} F\left(T_{i}\right)$ such that $\left\langle A x^{*}, J\left(x-x^{*}\right)\right\rangle \geq 0$, $\forall x \in \cap_{i=1}^{N} F\left(T_{i}\right)$,

and the following iteration process:

$$
\begin{aligned}
x_{0} & \in C, \\
z_{n} & =c_{n} x_{n}+\left(1-c_{n}\right) S x_{n}, \\
x_{n+1} & =Q_{C}\left[\left(1-\alpha_{n}\right)\left(\beta_{n} x_{n}+\left(1-\beta_{n}\right) Q_{C}[I-\gamma A] z_{n}\right)\right],
\end{aligned}
$$

where $S:=[(1-\mu) I+\mu T]$, for $T:=\theta_{1} T_{1}+\theta_{2} T_{2}+\cdots+$ $\theta_{n} T_{N}$, such that $\theta_{1}+\theta_{2}+\cdots+\theta_{N}=1,0<\mu<\mu_{0}=$ $\min \left\{1,\left(q \lambda / c_{q}\right)^{1 /(q-1)}\right\}$, for $\lambda:=\min \left\{\lambda_{i}: i=1,2, \ldots, N\right\}$, and $0<\gamma<\left(q \eta / c_{q}\right)^{1 /(q-1)}$, and $c_{q}$ is the real number in (16). In addition, we assume $\left\{\alpha_{n}\right\} \subset(0, c) \subset(0,1)$ and $\left\{\beta_{n}\right\},\left\{c_{n}\right\} \subset$ $[a, b] \subset(0,1)$ as real sequences satisfying the following control conditions: (i) $\lim _{n \rightarrow \infty} \alpha_{n}=0$, (ii) $\sum \alpha_{n}=\infty$, $\lim _{n \rightarrow \infty}\left(\left|\beta_{n}-\beta_{n-1}\right| / \alpha_{n-1}\right)=0, \lim _{n \rightarrow \infty}\left(\left|\alpha_{n}-\alpha_{n-1}\right| / \alpha_{n-1}\right)=$ 0 , and $\lim _{n \rightarrow \infty}\left(\left|c_{n}-c_{n-1}\right| / \alpha_{n-1}\right)=0$.

We now prove our main theorem.

Theorem 12. Let $C$ be a nonempty closed convex subset of a real uniformly convex and q-uniformly smooth Banach space $E$ possessing weakly sequentially continuous duality mapping. Let $T_{i}: C \rightarrow C$, for $i=1, \ldots, N$, be $\lambda_{i}$-strictly pseudocontractive mappings, and let $A: C \rightarrow E$ be $\eta$-inverse strongly accretive mapping. Let $\left\{x_{n}\right\}$ be a sequence defined by (25). Assume that $\mathscr{F}:=F \cap \operatorname{VI}(C, A) \neq \emptyset$, where $F=\cap_{i=1}^{N} F\left(T_{i}\right)=F(S)$. Then, $\left\{x_{n}\right\}$ converges strongly to $Q_{\mathscr{F}}(0)$, where $Q_{\mathscr{F}}$ is a sunny nonexpansive retraction of $E$ onto $\mathscr{F}$, which is a solution of the variational inequality (24).

Proof. By Lemmas 7 and 8 we have that $S$ is nonexpansive. In addition, by Lemma 9 we get that $Q_{C}[I-\gamma A]$ is nonexpansive. Let $p \in \mathscr{F}$ and, let $y_{n}:=\beta_{n} x_{n}+\left(1-\beta_{n}\right) Q_{C}[I-\gamma A] z_{n}$. Then from (25), Lemmas 8 and 9 we have that

$$
\begin{aligned}
\left\|z_{n}-p\right\| \leq & c_{n}\left\|x_{n}-p\right\|+\left(1-c_{n}\right)\left\|S x_{n}-p\right\| \\
\leq & \beta_{n}\left\|x_{n}-p\right\|+\left(1-\beta_{n}\right)\left\|x_{n}-p\right\| \\
\leq & \left\|x_{n}-p\right\|, \\
\left\|y_{n}-p\right\| \leq & \beta_{n}\left\|x_{n}-p\right\| \\
& +\left(1-\beta_{n}\right)\left\|Q_{C}[I-\gamma A] z_{n}-Q_{C}[p-\gamma A p]\right\| \\
\leq & \beta_{n}\left\|x_{n}-p\right\|+\left(1-\beta_{n}\right)\left\|z_{n}-p\right\| \\
\leq & \beta_{n}\left\|x_{n}-p\right\|+\left(1-\beta_{n}\right)\left\|x_{n}-p\right\| \\
\leq & \left\|x_{n}-p\right\| .
\end{aligned}
$$

Thus, from (25) and (27), we get that

$$
\begin{aligned}
\left\|x_{n+1}-p\right\| & =\left\|Q_{C}\left[\left(1-\alpha_{n}\right) y_{n}\right]-Q_{C} p\right\| \\
& \leq\left(1-\alpha_{n}\right)\left\|y_{n}-p\right\|+\alpha_{n}\|p\| \\
& \leq\left(1-\alpha_{n}\right)\left\|x_{n}-p\right\|+\alpha_{n}\|p\| .
\end{aligned}
$$

Therefore, by induction,

$$
\left\|x_{n+1}-p\right\| \leq \max \left\{\left\|x_{0}-p\right\|,\|p\|\right\}, \quad \forall n \geq 0,
$$

which implies that $\left\{x_{n}\right\}$ and hence $\left\{y_{n}\right\},\left\{z_{n}\right\}$, and $\left\{A x_{n}\right\}$ are bounded. Furthermore, from (25), we obtain that

$$
\begin{aligned}
& \left\|z_{n+1}-z_{n}\right\| \\
& =\| c_{n+1} x_{n+1}+\left(1-c_{n+1}\right) S x_{n+1} \\
& -\left(c_{n} x_{n}+\left(1-c_{n}\right) S x_{n}\right) \| \\
& =\left\|\left(c_{n+1} x_{n+1}-c_{n} x_{n}\right)+\left(1-c_{n+1}\right) S x_{n+1}-\left(1-c_{n}\right) S x_{n}\right\| \\
& \leq\left|c_{n+1}-c_{n}\right|\left\|x_{n+1}\right\|+c_{n}\left\|x_{n+1}-x_{n}\right\| \\
& +\left|c_{n+1}-c_{n}\right|\left\|S x_{n+1}\right\|+\left(1-c_{n}\right)\left\|x_{n+1}-x_{n}\right\| \\
& \leq\left\|x_{n+1}-x_{n}\right\|+\left|c_{n+1}-c_{n}\right|\left[\left\|x_{n+1}\right\|+\left\|S x_{n+1}\right\|\right] \text {, }
\end{aligned}
$$

$$
\begin{aligned}
\| y_{n+1}- & y_{n} \| \\
= & \| \beta_{n+1} x_{n+1}+\left(1-\beta_{n+1}\right) Q_{C}\left[z_{n+1}-\gamma A z_{n+1}\right] \\
& -\left(\beta_{n} x_{n}+\left(1-\beta_{n}\right) Q_{C}\left[z_{n}-\gamma A z_{n}\right]\right) \| \\
= & \|\left(\beta_{n+1} x_{n+1}-\beta_{n} x_{n}\right)+\left(1-\beta_{n+1}\right) \\
& \times Q_{C}\left[z_{n+1}-\gamma A z_{n+1}\right]-\left(1-\beta_{n}\right) Q_{C}\left[z_{n}-\gamma A z_{n}\right] \| \\
\leq & \left|\beta_{n+1}-\beta_{n}\right|\left\|x_{n+1}\right\|+\beta_{n}\left\|x_{n+1}-x_{n}\right\| \\
& +\left|\beta_{n+1}-\beta_{n}\right|\left\|Q_{C}\left[z_{n+1}-\gamma A z_{n+1}\right]\right\|+\left(1-\beta_{n}\right) \\
& \times \\
\leq & \beta_{n}\left\|z_{n+1}-z_{n}\right\| \\
& +\mid \beta_{n+1}-x_{n} \| \\
& +\left(1-\beta_{n}\right) \|\left[\left\|x_{n+1}\right\|+\left\|z_{n+1}-z_{n}\right\|\right. \\
\leq & \beta_{n}\left\|x_{n+1}-x_{n}\right\| \\
& \left.+\left|\beta_{n+1}-\beta_{n}\right|\left[\left\|x_{n+1}\right\|+\left\|Q_{C}\left[z_{n+1}-\gamma A z_{n+1}\right]\right\|\right] \|\right] \\
& +\left(1-\beta_{n}\right)\left\|x_{n+1}-x_{n}\right\| \\
& +\left|c_{n+1}-c_{n}\right|\left[\left\|x_{n+1}\right\|+\left\|S x_{n+1}\right\|\right] \\
& \times\left[\left\|x_{n+1}-x_{n}\right\|+\left|\beta_{n+1}-\beta_{n}\right|\right. \\
& \left.+\left\|c_{C+1}-c_{n} \mid\left[z_{n+1}-\gamma A z_{n+1}\right]\right\|\right] \\
& \\
&
\end{aligned}
$$


And, hence, from (25) and (31), we have that

$$
\begin{aligned}
\| x_{n+1}- & x_{n} \| \\
= & \left\|Q_{C}\left[\left(1-\alpha_{n}\right) y_{n}\right]-Q_{C}\left[\left(1-\alpha_{n-1}\right) y_{n-1}\right]\right\| \\
\leq & \left\|\left(1-\alpha_{n}\right) y_{n}-\left(1-\alpha_{n-1}\right) y_{n-1}\right\| \\
\leq & \|\left(1-\alpha_{n}\right) y_{n}-\left(1-\alpha_{n-1}\right) y_{n} \\
& +\left(1-\alpha_{n-1}\right) y_{n}-\left(1-\alpha_{n-1}\right) y_{n-1} \| \\
\leq & \left|\alpha_{n}-\alpha_{n-1}\right|\left\|y_{n}\right\|+\left(1-\alpha_{n-1}\right)\left\|y_{n-1}-y_{n}\right\| \\
\leq & \left(1-\alpha_{n-1}\right)\left\|x_{n}-x_{n-1}\right\|+\left|\alpha_{n}-\alpha_{n-1}\right| M \\
& +\left|\beta_{n}-\beta_{n-1}\right| M+\left|c_{n}-c_{n-1}\right| M,
\end{aligned}
$$

for some $M>0$. Thus, using the properties of $\left\{\alpha_{n}\right\},\left\{\beta_{n}\right\},\left\{c_{n}\right\}$, (32), and Lemma 2 , we obtain that $\left\|x_{n+1}-x_{n}\right\| \rightarrow 0$, as $n \rightarrow$ $\infty$, which implies from (31) that $\left\|y_{n+1}-y_{n}\right\| \rightarrow 0$, as $n \rightarrow \infty$. Again from (25), we have that $\left\|x_{n+1}-y_{n}\right\|=\| Q_{C}\left[\left(1-\alpha_{n}\right) y_{n}\right]-$ $Q_{C} y_{n}\left\|=\alpha_{n}\right\| y_{n} \| \rightarrow 0$, as $n \rightarrow \infty$. Consequently,

$$
\left\|x_{n}-y_{n}\right\| \longrightarrow 0, \quad \text { as } n \longrightarrow \infty \text {. }
$$

Now, we prove that $\left\{x_{n}\right\}$ converges strongly to the point $x^{*}=Q_{\mathscr{F}}(0)$. Let $t_{n}=Q_{C}[I-\gamma A] z_{n}$, and let $d_{n}=\left(1-\alpha_{n}\right) y_{n}$. Then, since $\alpha_{n} \rightarrow 0$, we have that

$$
\left\|d_{n}-y_{n}\right\|=\alpha_{n}\left\|y_{n}\right\| \longrightarrow 0, \quad \text { as } n \longrightarrow \infty \text {. }
$$

Furthermore, from (25), Lemma 5, and Lemma 10, we get that

$$
\begin{aligned}
\| x_{n+1}- & x^{*} \|^{2} \\
= & \left\|Q_{C}\left[\left(1-\alpha_{n}\right) y_{n}\right]-Q_{C} x^{*}\right\|^{2} \\
\leq & \left\|\alpha_{n}\left(-x^{*}\right)+\left(1-\alpha_{n}\right)\left(y_{n}-x^{*}\right)\right\|^{2} \\
\leq & \left(1-\alpha_{n}\right)\left\|y_{n}-x^{*}\right\|^{2}-2 \alpha_{n}\left\langle x^{*}, j\left(d_{n}-x^{*}\right)\right\rangle \\
\leq & \left(1-\alpha_{n}\right)\left[\beta_{n}\left\|x_{n}-x^{*}\right\|^{2}+\left(1-\beta_{n}\right)\left\|t_{n}-x^{*}\right\|^{2}\right. \\
& \left.\quad-\beta_{n}\left(1-\beta_{n}\right) g\left(\left\|t_{n}-x_{n}\right\|\right)\right] \\
& -2 \alpha_{n}\left\langle x^{*}, j\left(d_{n}-x^{*}\right)\right\rangle \\
\leq & \left(1-\alpha_{n}\right) \beta_{n}\left\|x_{n}-x^{*}\right\|^{2}+\left(1-\alpha_{n}\right)\left(1-\beta_{n}\right)\left\|z_{n}-x^{*}\right\|^{2} \\
& -\beta_{n}\left(1-\beta_{n}\right)\left(1-\alpha_{n}\right) g\left(\left\|t_{n}-x_{n}\right\|\right) \\
& -2 \alpha_{n}\left\langle x^{*}, j\left(d_{n}-x^{*}\right)\right\rangle
\end{aligned}
$$

$$
\begin{aligned}
\leq & \left(1-\alpha_{n}\right) \beta_{n}\left\|x_{n}-x^{*}\right\|^{2}+\left(1-\alpha_{n}\right)\left(1-\beta_{n}\right) \\
\times & {\left[c_{n}\left\|x_{n}-p\right\|^{2}+\left(1-c_{n}\right)\left\|S x_{n}-p\right\|^{2}\right.} \\
& \left.-c_{n}\left(1-c_{n}\right) g\left(\left\|S x_{n}-x_{n}\right\|\right)\right] \\
- & \beta_{n}\left(1-\beta_{n}\right)\left(1-\alpha_{n}\right) g\left(\left\|t_{n}-x_{n}\right\|\right) \\
- & 2 \alpha_{n}\left\langle x^{*}, j\left(d_{n}-x^{*}\right)\right\rangle \\
\leq & \left(1-\alpha_{n}\right) \beta_{n}\left\|x_{n}-x^{*}\right\|^{2}+\left(1-\alpha_{n}\right)\left(1-\beta_{n}\right) \\
\times & {\left[c_{n}\left\|x_{n}-p\right\|^{2}+\left(1-c_{n}\right)\left\|S x_{n}-p\right\|^{2}\right.} \\
& \left.-c_{n}\left(1-c_{n}\right) g\left(\left\|S x_{n}-x_{n}\right\|\right)\right] \\
- & \beta_{n}\left(1-\beta_{n}\right)\left(1-\alpha_{n}\right) g\left(\left\|t_{n}-x_{n}\right\|\right) \\
- & 2 \alpha_{n}\left\langle x^{*}, j\left(d_{n}-x^{*}\right)\right\rangle,
\end{aligned}
$$

which implies that

$$
\begin{aligned}
\| x_{n+1}- & x^{*} \|^{2} \\
\leq & \left(1-\alpha_{n}\right)\left\|x_{n}-x^{*}\right\|^{2}-c_{n}\left(1-c_{n}\right)\left(1-\alpha_{n}\right)\left(1-\beta_{n}\right) \\
& \times g\left(\left\|S x_{n}-x_{n}\right\|\right) \\
& -\beta_{n}\left(1-\beta_{n}\right)\left(1-\alpha_{n}\right) g\left(\left\|t_{n}-x_{n}\right\|\right) \\
& -2 \alpha_{n}\left\langle x^{*}, j\left(d_{n}-x^{*}\right)\right\rangle \\
\leq & \left(1-\alpha_{n}\right)\left\|x_{n}-x^{*}\right\|^{2}-2 \alpha_{n}\left\langle x^{*}, j\left(d_{n}-x^{*}\right)\right\rangle
\end{aligned}
$$

Now, following the method of proof of Lemma 3.2 of Maingé [5], we consider two cases.

Case 1. Suppose that there exists $n_{0} \in \mathbb{N}$ such that $\left\{\left\|x_{n}-x^{*}\right\|\right\}$ is decreasing for all $n \geq n_{0}$. Then, we get that $\left\{\left\|x_{n}-x^{*}\right\|\right\}$ is convergent. Thus, from (36) and the fact that $\alpha_{n} \rightarrow 0$, as $n \rightarrow \infty$, we have that

$$
\begin{array}{r}
g\left(\left\|S x_{n}-x_{n}\right\|\right) \longrightarrow 0, \quad g\left(\left\|t_{n}-x_{n}\right\|\right) \\
\text { as } n \longrightarrow 0,
\end{array}
$$

which implies that

$$
\begin{gathered}
\left\|S x_{n}-x_{n}\right\| \longrightarrow 0, \\
\left\|t_{n}-x_{n}\right\|=\left\|Q_{C}[I-\gamma A] x_{n}-x_{n}\right\| \longrightarrow 0, \quad \text { as } n \longrightarrow \infty .
\end{gathered}
$$

In addition, since $\left\{d_{n}\right\}$ is bounded subset of a reflexive space $E$, we can choose a subsequence $\left\{d_{n_{i}}\right\}$ of $\left\{d_{n}\right\}$ such that $d_{n_{i}} \rightarrow z$ and $\lim \sup _{n \rightarrow \infty}\left\langle x^{*}, j\left(d_{n}-x^{*}\right)\right\rangle=$ $\lim _{i \rightarrow \infty}\left\langle x^{*}, j\left(d_{n_{i}}-x^{*}\right)\right\rangle$. This implies from (34) and (33) that $x_{n_{i}} \rightarrow z$. Then, from (39) and Lemma 4, we have that $z \in F(S)=\cap_{i=1}^{N} F\left(T_{i}\right)$. Moreover, from (39) and Lemma 4, we have that $z \in F\left(Q_{C}[I-\gamma A]\right)$, and by Lemma 3, we get 
$z \in V I(C, A)$, and hence $z \in \mathscr{F}$. Therefore, using the fact that $E$ has a weakly sequentially continuous duality mapping and Lemma 1, we immediately obtain that

$$
\begin{aligned}
\limsup _{n \rightarrow \infty} & \left\langle x^{*}, j\left(d_{n}-x^{*}\right)\right\rangle \\
& =\lim _{i \rightarrow \infty}\left\langle x^{*}, j\left(d_{n_{i}}-x^{*}\right)\right\rangle \\
& =\left\langle x^{*}, j\left(z-x^{*}\right)\right\rangle \geq 0 .
\end{aligned}
$$

Then, it follows from (37), (40), and Lemma 2 that $\| x_{n}-$ $x^{*} \| \rightarrow 0$, as $n \rightarrow \infty$. Consequently, $x_{n} \rightarrow x^{*}=Q_{\mathscr{F}} 0$.

Case 2. Suppose that there exists a subsequence $\left\{n_{i}\right\}$ of $\{n\}$ such that

$$
\left\|x_{n_{i}}-x^{*}\right\|<\left\|x_{n_{i}+1}-x^{*}\right\|
$$

for all $i \in \mathbb{N}$. Then, by Lemma 11, there exists a nondecreasing sequence $\left\{m_{k}\right\} \subset \mathbb{N}$ such that $m_{k} \rightarrow \infty$, and

$$
\begin{gathered}
\left\|x_{m_{k}}-x^{*}\right\| \leq\left\|x_{m_{k}+1}-x^{*}\right\|, \\
\left\|x_{k}-x^{*}\right\| \leq\left\|x_{m_{k}+1}-x^{*}\right\|,
\end{gathered}
$$

for all $k \in \mathbb{N}$. Now, from (36) and the fact that $\alpha_{n} \rightarrow 0$, we get that $x_{m_{k}}-S x_{m_{k}} \rightarrow 0$ and $\left\|Q_{C}[I-\gamma A] x_{m_{k}}-x_{m_{k}}\right\| \rightarrow 0$, as $k \rightarrow \infty$. Thus, like in Case 1 , we obtain that

$$
\limsup _{k \rightarrow \infty}\left\langle x^{*}, j\left(d_{m_{k}}-x^{*}\right)\right\rangle \geq 0
$$

Moreover, from (37), we have that

$$
\begin{aligned}
& \left\|x_{m_{k}+1}-x^{*}\right\|^{2} \\
& \quad \leq\left(1-\alpha_{m_{k}}\right)\left\|x_{m_{k}}-x^{*}\right\|^{2}-2 \alpha_{m_{k}}\left\langle x^{*}, j\left(d_{m_{k}}-x^{*}\right)\right\rangle,
\end{aligned}
$$

which implies from (42) and (44) that

$$
\begin{aligned}
\alpha_{m_{k}} \| & x_{m_{k}}-x^{*} \|^{2} \\
\leq & \left\|x_{m_{k}}-x^{*}\right\|^{2}-\left\|x_{m_{k}+1}-x^{*}\right\|^{2} \\
& \quad-2 \alpha_{m_{k}}\left\langle x^{*}, j\left(d_{m_{k}}-x^{*}\right)\right\rangle \\
\leq & -2 \alpha_{m_{k}}\left\langle x^{*}, j\left(d_{m_{k}}-x^{*}\right)\right\rangle .
\end{aligned}
$$

Now, since $\alpha_{m_{k}}>0$, we obtain that

$$
\left\|x_{m_{k}}-x^{*}\right\|^{2} \leq-2\left\langle x^{*}, j\left(d_{m_{k}}-x^{*}\right)\right\rangle
$$

and using (43), we get that $\left\|x_{m_{k}}-x^{*}\right\| \rightarrow 0$. This together with (44) implies that $\left\|x_{m_{k}+1}-x^{*}\right\| \rightarrow 0$, as $k \rightarrow \infty$. But $\left\|x_{k}-x^{*}\right\| \leq\left\|x_{m_{k}+1}-x^{*}\right\|$, for all $k \in \mathbb{N}$; thus, we obtain that $x_{k} \rightarrow x^{*}$. Therefore, from both cases, we can conclude that $\left\{x_{n}\right\}$ converges strongly to $x^{*}=P_{\mathscr{F}}(0)$, which is a solution of the variational inequality (24), and the proof is complete.
If in Theorem 12, we consider that $N=1$, we get the following corollary.

Corollary 13. Let $C$ be a nonempty closed convex subset of a real uniformly convex and q-uniformly smooth Banach space $E$ possessing weakly sequentially continuous duality mapping. Let $T: C \rightarrow C$ be a $\lambda$-strictly pseudocontractive mapping, and let $A: C \rightarrow E$ be an $\eta$-inverse strongly accretive mapping. Let $\left\{x_{n}\right\}$ be a sequence defined by (25), where $S:=$ $[(1-\mu) I+\mu T]$. Assume that $F:=F(T) \cap V I(C, A) \neq \emptyset$. Then, $\left\{x_{n}\right\}$ converges strongly to $Q_{F}(0)$ which is a solution of the variational inequality

Find $x^{*} \in F(T)$ such that $\left\langle A x^{*}, J\left(x-x^{*}\right)\right\rangle \geq 0$,

$$
\forall x \in F(T) .
$$

If in Theorem 12, we assume that $T_{i}$, for $i=1,2, \ldots, N$, are nonexpansive, we get the following corollary.

Corollary 14. Let $C$ be a nonempty closed convex subset of a real uniformly convex and q-uniformly smooth Banach space $E$ possessing weakly sequentially continuous duality mapping. Let $T_{i}: C \rightarrow C$, for $i=1,2, \ldots, N$, be nonexpansive mappings, and let $A: C \rightarrow E$ be an $\eta$-inverse strongly accretive mapping. For $x_{0} \in C$, let the sequence $\left\{x_{n}\right\}$ be generated iteratively by

$$
\begin{aligned}
z_{n} & =c_{n} x_{n}+\left(1-c_{n}\right) T x_{n}, \\
x_{n+1} & =Q_{C}\left[\left(1-\alpha_{n}\right)\left(\beta_{n} x_{n}+\left(1-\beta_{n}\right) Q_{C}[I-\gamma A] z_{n}\right)\right],
\end{aligned}
$$

where $T:=\theta_{0} I+\theta_{1} T_{1}+\cdots+\theta_{N} T_{N}$ for $\left\{\theta_{i}\right\}_{i=1}^{N},\left\{\alpha_{n}\right\},\left\{\beta_{n}\right\}$, $\gamma$ are as in (24). Assume that $\mathscr{F}:=F \cap \operatorname{VI}(C, A) \neq \emptyset$, where $F:=\cap_{i=1}^{N} F\left(T_{i}\right)=F(T)$. Then, $\left\{x_{n}\right\}$ converges strongly to $Q_{\mathscr{F}}(0)$, which is a solution of the variational inequality problem (24).

Proof. Lemma 6 and the method of proof of Theorem 12 provide the required assertion.

If in Corollary 14, we consider that $N=1$, we get the following corollary.

Corollary 15. Let $C$ be a nonempty closed convex subset of a real uniformly convex and q-uniformly smooth Banach space $E$ possessing weakly sequentially continuous duality mapping. Let $T: C \rightarrow C$ be a nonexpansive mapping, and let $A: C \rightarrow E$ be an $\eta$-inverse strongly accretive mapping. For $x_{0} \in C$, let the sequence $\left\{x_{n}\right\}$ be generated iteratively by

$$
\begin{aligned}
z_{n} & =c_{n} x_{n}+\left(1-c_{n}\right) T x_{n}, \\
x_{n+1} & =Q_{C}\left[\left(1-\alpha_{n}\right)\left(\beta_{n} x_{n}+\left(1-\beta_{n}\right) Q_{C}[I-\gamma A] z_{n}\right)\right] .
\end{aligned}
$$

Assume that $F:=F(T) \cap \operatorname{VI}(C, A) \neq \emptyset$. Then, $\left\{x_{n}\right\}$ converges strongly to $Q_{F}(0)$, which is a solution of the variational inequality problem

$$
\begin{aligned}
& \text { Find } x^{*} \in F(T) \text { such that }\left\langle A x^{*}, J\left(x-x^{*}\right)\right\rangle \geq 0 \text {, } \\
& \forall x \in F(T) \text {. }
\end{aligned}
$$


If in Corollary 14, we assume that $T=T_{1}=T_{2}=\cdots=$ $T_{N}=I$, we obtain the following corollary.

Corollary 16. Let $C$ be a nonempty closed convex subset of a real uniformly convex and q-uniformly smooth Banach space E possessing weakly sequentially continuous duality mapping. Let $A: C \rightarrow E$ be an $\eta$-inverse strongly accretive mapping. For $x_{0} \in C$, let the sequence $\left\{x_{n}\right\}$ be generated iteratively by

$$
x_{n+1}=Q_{C}\left[\left(1-\alpha_{n}\right)\left(\beta_{n} x_{n}+\left(1-\beta_{n}\right) Q_{C}[I-\gamma A] x_{n}\right)\right] \text {. }
$$

Assume that $\operatorname{VI}(C, A) \neq \emptyset$. Then, $\left\{x_{n}\right\}$ converges strongly to $Q_{V I(C, A)}(0)$, where $Q_{V I(C, A)}$ is a sunny nonexpansive retraction of $E$ onto $V I(C, A)$.

If in Theorem 12, we assume that $A$ is an $\alpha$-strongly accretive and $L$-Lipschitzian continuous mapping, we obtain the following corollary.

Corollary 17. Let $C$ be a nonempty closed convex subset of a real uniformly convex and q-uniformly smooth Banach space E possessing weakly sequentially continuous duality mapping. Let $T_{i}: C \rightarrow C$, for $i=1, \ldots, N$, be $\lambda_{i}$-strictly pseudocontractive mappings, and Let $A: C \rightarrow E$ be an $\alpha$ strongly accretive and L-Lipschitzian continuous mapping. Let $\left\{x_{n}\right\}$ be a sequence defined by (25) for $\eta=\alpha / L^{2}$. Assume that $\mathscr{F}:=F \cap \operatorname{VI}(C, A) \neq \emptyset$, where $F:=\cap_{i=1}^{N} F\left(T_{i}\right)=F(S)$. Then, $\left\{x_{n}\right\}$ converges strongly to $Q_{\mathscr{F}}(0)$, which is a solution of the variational inequality problem

$$
\begin{array}{r}
\text { Find } x^{*} \in \cap_{i=1}^{N} F\left(T_{i}\right) \text { such that }\left\langle A x^{*}, J\left(x-x^{*}\right)\right\rangle \geq 0, \\
\forall x \in \cap_{i=1}^{N} F\left(T_{i}\right) .
\end{array}
$$

Proof. We note that if $A$ is an $\alpha$-strongly accretive and $L$ Lipschitzian continuous mapping of $C$ into $E$, then we have that

$$
\begin{array}{r}
\langle A x-A y, j(x-y)\rangle \geq \alpha\|x-y\|^{2} \geq \frac{\alpha}{L^{2}}\|A x-A y\|^{2}, \\
\forall x, y \in C,
\end{array}
$$

and hence, $A$ is an $\eta$-inverse strongly accretive mapping with $\eta=\alpha / L^{2}$. Thus, the conclusion follows from Theorem 12 .

If $E=H$, a real Hilbert space, then $E$ is a uniformly convex and $q$-uniformly smooth Banach space $E$ for $1<$ $q<\infty$ possessing weakly sequentially continuous duality mapping. In this case, we have that $Q_{C}=P_{C}$, projection mapping from $H$ onto $C$. Thus, we have the following corollary.

Corollary 18. Let $C$ be a nonempty closed convex subset of a real Hilbert space $H$. Let $T_{i}: C \rightarrow C$, for $i=1, \ldots, N$, be $\lambda_{i}$-strictly pseudocontractive mappings, and let $A: C \rightarrow E$ be an $\eta$-inverse strongly accretive mapping. For $x_{0} \in C$, let the sequence $\left\{x_{n}\right\}$ be generated iteratively by

$$
\begin{aligned}
& z_{n}=c_{n} x_{n}+\left(1-c_{n}\right) S x_{n}, \\
& x_{n+1}=P_{C}\left[\left(1-\alpha_{n}\right)\left(\beta_{n} x_{n}+\left(1-\beta_{n}\right) P_{C}[I-\gamma A] z_{n}\right)\right],
\end{aligned}
$$

where $S:=[(1-\mu) I+\mu T]$, for $T:=\theta_{1} T_{1}+\theta_{2} T_{2}+\cdots+\theta_{n} T_{N}$, such that $\theta_{1}+\theta_{2}+\cdots+\theta_{N}=1,0<\mu<\min \{1,2 \lambda\}$, for $\lambda:=\min \left\{\lambda_{i}: i=1,2, \ldots, N\right\}$, and $0<\gamma<2 \eta$. Assume that $\mathscr{F}:=F \cap \operatorname{VI}(C, A) \neq \emptyset$, where $F=\cap_{i=1}^{N} F\left(T_{i}\right)=F(S)$. Then, $\left\{x_{n}\right\}$ converges strongly to $P_{\mathscr{F}}(0)$, which is a solution of the variational inequality

$$
\begin{array}{r}
\text { Find } x^{*} \in \cap_{i=1}^{N} F\left(T_{i}\right) \text { such that }\left\langle A x^{*}, x-x^{*}\right\rangle \geq 0, \\
\qquad \forall x \in \cap_{i=1}^{N} F\left(T_{i}\right) .
\end{array}
$$

Remark 19. Theorem 12 complements Theorem 3.2 of Yao et al. [8] in more general Banach spaces for $\eta$-inverse strongly accretive mappings. Moreover, Theorem 12 improves Theorem 3.1 of Aoyama et al. [13] and Theorem 3.7 of Saejung et al. [23] in the sense that our convergence is strong in the set of common fixed points of finite family of strictly pseudocontractive mappings.

\section{References}

[1] R. P. Agarwal, D. ORegan, and D. R. Sahu, Fixed Point Theory for Lipschitzian-Type Mappings with Applications, Springer, New York, NY, USA, 2000.

[2] G. Stampacchia, "Formes bilinéaires coercitives sur les ensembles convexes," Comptes Rendus de l'Académie des Sciences, vol. 258, pp. 4413-4416, 1964.

[3] H. Iiduka and W. Takahashi, "Strong convergence theorems for nonexpansive mappings and inverse-strongly monotone mappings," Nonlinear Analysis. Theory, Methods \& Applications, vol. 61, no. 3, pp. 341-350, 2005.

[4] G. M. Korpelevič, "An extragradient method for finding saddle points and for other problems," Ekonomika i Matematicheskie Metody, vol. 12, no. 4, pp. 747-756, 1976.

[5] P.-E. Maingé, "Strong convergence of projected subgradient methods for nonsmooth and nonstrictly convex minimization," Set-Valued Analysis, vol. 16, no. 7-8, pp. 899-912, 2008.

[6] M. A. Noor, "A class of new iterative methods for general mixed variational inequalities," Mathematical and Computer Modelling, vol. 31, no. 13, pp. 11-19, 2000.

[7] I. Yamada, “The hybrid steepest descent method for the variational inequality problem over the intersection of fixed point sets of nonexpansive mappings," in Inherently parallel algorithms in feasibility and optimization and their applications, vol. 8, pp. 473-504, North-Holland, Amsterdam, The Netherlands, 2001.

[8] Y. Yao, Y.-C. Liou, C.-L. Li, and H.-T. Lin, "Extended extragradient methods for generalized variational inequalities," Journal of Applied Mathematics, vol. 2012, Article ID 237083, 14 pages, 2012.

[9] Y. Yao and H.-K. Xu, "Iterative methods for finding minimumnorm fixed points of nonexpansive mappings with applications," Optimization, vol. 60, no. 6, pp. 645-658, 2011.

[10] H. Zegeye, E. U. Ofoedu, and N. Shahzad, "Convergence theorems for equilibrium problem, variational inequality problem and countably infinite relatively quasi-nonexpansive mappings," Applied Mathematics and Computation, vol. 216, no. 12, pp. 3439-3449, 2010.

[11] H. Zegeye and N. Shahzad, "A hybrid scheme for finite families of equilibrium, variational inequality and fixed point problems," 
Nonlinear Analysis. Theory, Methods \& Applications, vol. 74, no. 1, pp. 263-272, 2011.

[12] H. Zegeye and N. Shahzad, "Strong convergence theorems for a common zero of a countably infinite family of $\alpha$-inverse strongly accretive mappings," Nonlinear Analysis. Theory, Methods \& Applications, vol. 71, no. 1-2, pp. 531-538, 2009.

[13] K. Aoyama, H. Iiduka, and W. Takahashi, "Weak convergence of an iterative sequence for accretive operators in Banach spaces," Fixed Point Theory and Applications, vol. 2006, Article ID 35390, 2006.

[14] F. E. Browder and W. V. Petryshyn, "Construction of fixed points of nonlinear mappings in Hilbert space," Journal of Mathematical Analysis and Applications, vol. 20, pp. 197-228, 1967.

[15] H. K. Xu, "Inequalities in Banach spaces with applications," Nonlinear Analysis. Theory, Methods \& Applications, vol. 16, no. 12, pp. 1127-1138, 1991.

[16] Y. I. Alber, A. N. Iusem, and M. V. Solodov, "Minimization of nonsmooth convex functionals in Banach spaces," Journal of Convex Analysis, vol. 4, no. 2, pp. 235-254, 1997.

[17] F. E. Browder, "Nonlinear Operators and nonlinear equations of evolution in Banach spaces," in Nonlinear Functional Analysis, pp. 1-308, American Mathematical scociety, Rhode Island, New England, 1976.

[18] H.-K. Xu, "Iterative algorithms for nonlinear operators," Journal of the London Mathematical Society, vol. 66, no. 1, pp. 240-256, 2002.

[19] C. H. Morales and J. S. Jung, "Convergence of paths for pseudocontractive mappings in Banach spaces," Proceedings of the American Mathematical Society, vol. 128, no. 11, pp. 3411$3419,2000$.

[20] C. E. Chidume, H. Zegeye, and N. Shahzad, "Convergence theorems for a common fixed point of a finite family of nonself nonexpansive mappings," Fixed Point Theory and Applications, no. 2, pp. 233-241, 2005.

[21] Y. Zhang and Y. Guo, "Weak convergence theorems of three iterative methods for strictly pseudocontractive mappings of Browder-Petryshyn type," Fixed Point Theory and Applications, vol. 2008, Article ID 672301, 13 pages, 2008.

[22] H. Zhang and Y. Su, "Strong convergence theorems for strict pseudo-contractions in q-uniformly smooth Banach spaces," Nonlinear Analysis. Theory, Methods \& Applications, vol. 70, no. 9, pp. 3236-3242, 2009.

[23] S. Saejung, K. Wongchan, and P. Yotkaew, "Another weak convergence theorems for accretive mappings in Banach spaces," Fixed Point Theory and Applications, vol. 2011, article 26, 2011, http://www.fixedpointtheoryandapplications.com/ content/2011/1/26. 


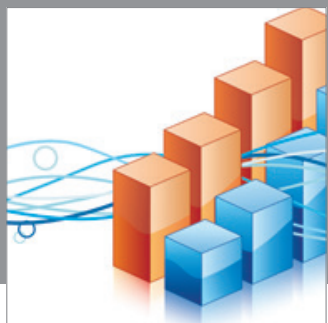

Advances in

Operations Research

mansans

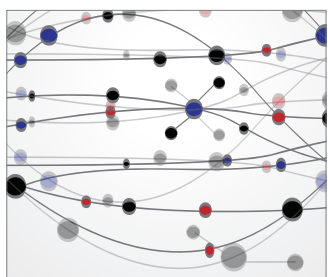

The Scientific World Journal
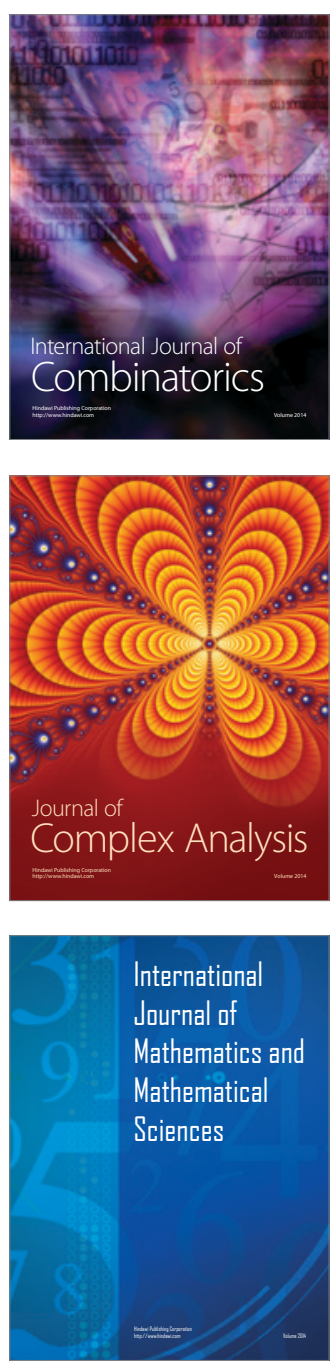
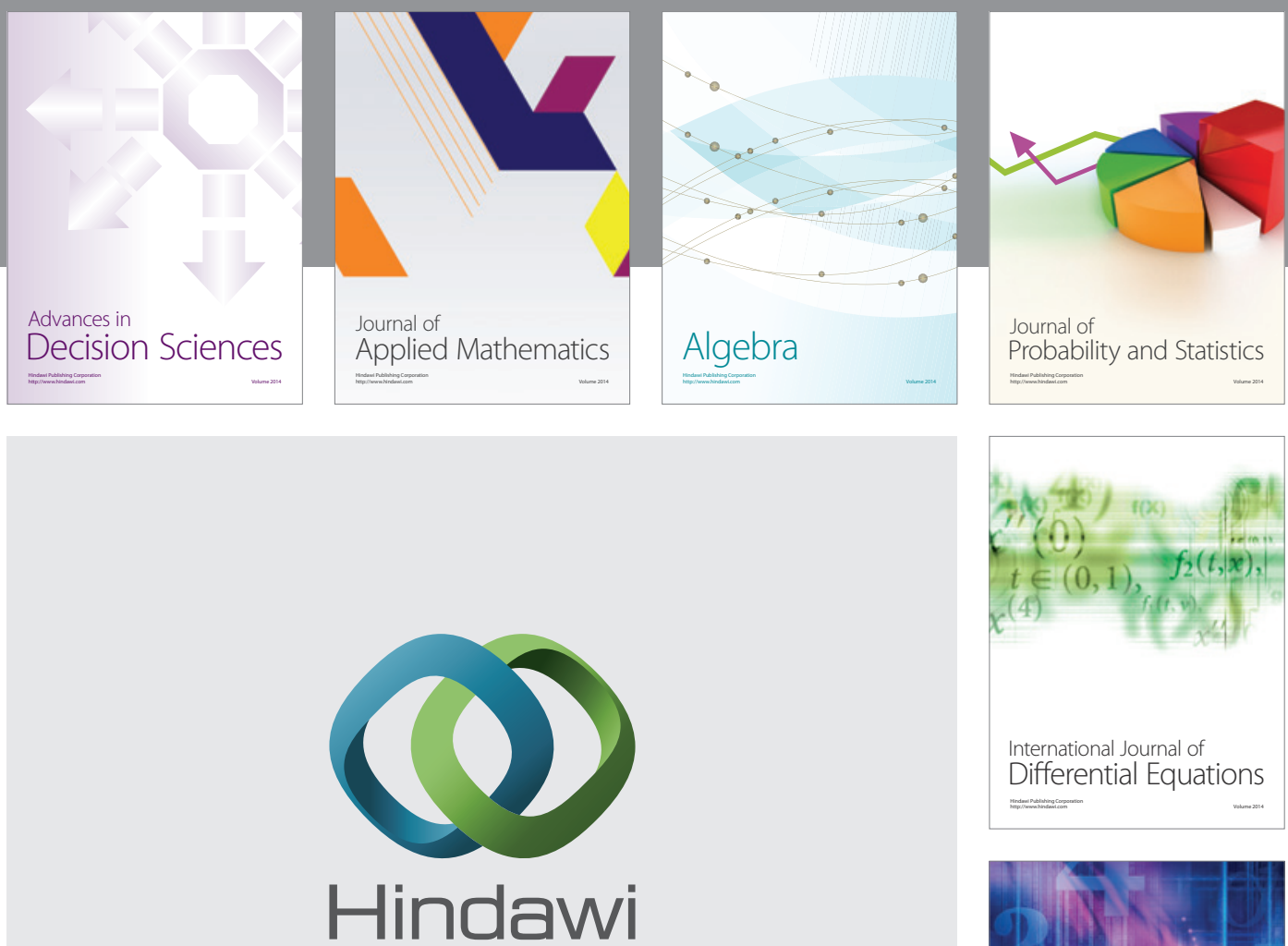

Submit your manuscripts at http://www.hindawi.com
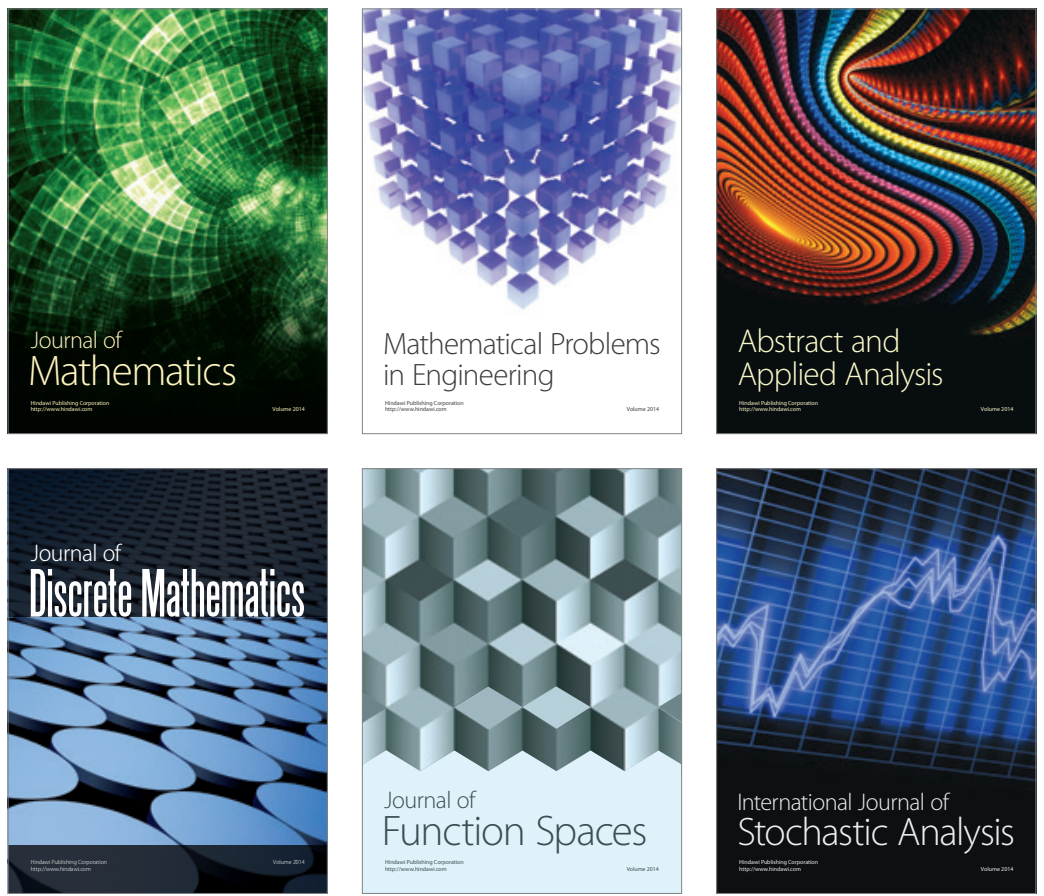

Journal of

Function Spaces

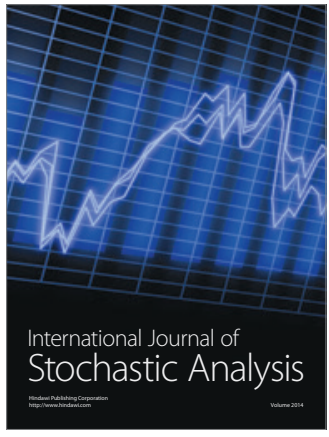

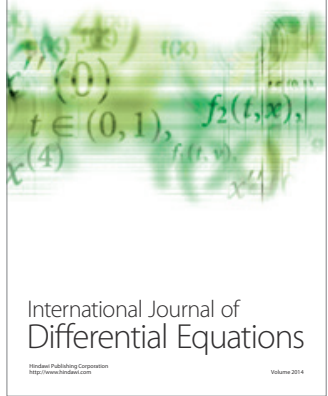
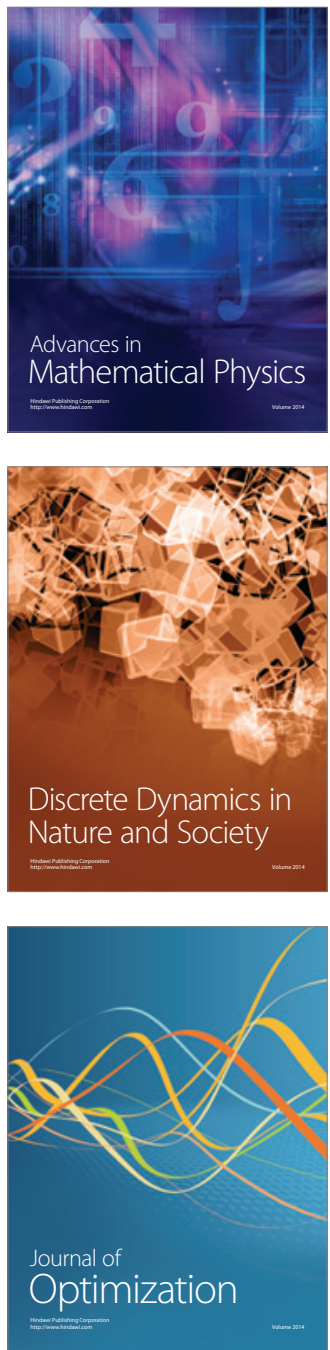